PROCEEDINGS OF THE

AMERICAN MATHEMATICAL SOCIETY

Volume 128, Number 5, Pages 1427-1432

S 0002-9939(99)05295-8

Article electronically published on October 27, 1999

\title{
ON EXACT QUADRATURE FORMULAS FOR HARMONIC FUNCTIONS ON POLYHEDRA
}

\author{
BJÖRN GUSTAFSSON AND MIHAI PUTINAR
}

(Communicated by David R. Larson)

\begin{abstract}
A classical quadrature result for analytic functions of a complex variable due to Motzkin and Schoenberg is extended to higher dimensions. A general scheme for integrating on polyhedra solutions of partial differential equations is discussed.
\end{abstract}

\section{INTRODUCTION}

A formula due to Motzkin-Schoenberg and later refined by Davis [1] asserts that the area integral on a triangle of the second derivative of an analytic function is a linear combination of its values at the vertices of the triangle. This fact was recently used in inverting the shape of a planar polygon from a finite sequence of its moments (see [2] and [3]). This note collects a few simple remarks which represent possible generalizations of the Motzkin-Schoenberg-Davis formula in higher dimensions.

The basis of all these formulas is of course Stokes' theorem. Our main result states that the integral of any three derivatives of a harmonic function on a polyhedron in $\mathbf{R}^{n}$ can be evaluated, with the loss of two derivatives, on the codimension-2 skeleton. This phenomenon is actually more general, and it persists for solutions of homogeneous partial differential operators of order two, for instance as long as the faces of the polyhedron are not characteristic. A better situation, when iterated integration is possible and the result can be pushed down to the 0-th skeleton, is in the case of pluriharmonic functions in $\mathbf{C}^{n}$ integated on cartesian products of planar polyhedra.

A variety of other remarkable quadrature formulas for harmonic functions integrated on polyhedra are known. However, in general the support of the representing measure is not contained in any level of the skeleton of the polyhedron (see [4] and 7]).

Notation. Below we denote by $P_{n}$ a bounded open polyhedron in $\mathbf{R}^{n}, n \geq 2$, by $P_{k}$ its $k$-dimensional skeleton, and by $d x^{k}$ the $k$-dimensional linear measure on $P_{k}$ induced from the (oriented) volume $n$-measure in $\mathbf{R}^{n}, 0 \leq k \leq n$. The partial derivatives will be denoted as $\partial_{j}=\frac{\partial}{\partial x_{j}}, 1 \leq j \leq n$. The dual $L^{*}$ of a differential operator $L$ is always considered with respect to the bilinear pairing between smooth functions of compact support and distributions.

Received by the editors July 2, 1998.

2000 Mathematics Subject Classification. Primary 65D32.

This research was partially supported by the National Science Foundation Grant DMS-9800666.

(C)2000 American Mathematical Society 


\section{MAin RESUlts}

To start with, we present a general formula for harmonic functions of $n$ variables.

Theorem 1. Let $P_{n}$ be a bounded polyhedron in $\mathbf{R}^{n}$ and let $L_{3}$ be a third order homogeneous partial differential operator with constant coefficients.

Then for each codimension-2 face $P_{n-2}{ }^{i}$ of $P_{n}, i \in I$, there exists a linear, first order, homogeneous differential operator with constant coefficients $L_{1}{ }^{i}$, such that:

$$
\int_{P_{n}} L_{3}(u) d x^{n}=\sum_{i \in I} \int_{P_{n-2}{ }^{i}} L_{1}{ }^{i}(u) d x^{n-2}
$$

for every harmonic function $u$ defined in a neighbourhood of $\overline{P_{n}}$.

Note that, since in case $n=2$ an analytic function is the gradient of a harmonic function, the above formula is equivalent (at least for simply connected polygons) to the Motzkin-Schoenberg-Davis formula; see [1] and [2].

A simple example shows that Theorem 1 is not valid for the skeleton of codimension 3. Indeed, take the standard simplex in $\mathbf{R}^{3}$ :

$$
P_{3}=\{(x, y, z) ; x+y+z \leq 1, x \geq 0, y \geq 0, z \geq 0\}
$$

and the harmonic function $u(x, y, z)=x y z$. Then the gradient of $u$ vanishes on all vertices of $P_{3}$, but

$$
\int_{P_{3}} \frac{\partial^{3} u(x, y, z)}{\partial x \partial y \partial z} d x d y d z \neq 0 .
$$

Formula (1) can be improved at least in two ways: by adapting the differential equation satisfied by $u$ to the polyhedron $P_{n}$, or by using direct products of polygons and a more restrictive class of functions.

In the first case, we state the following result.

Theorem 2. Let $P_{n}$ be a bounded polyhedron in $\mathbf{R}^{n}$, let $L_{m}$ be an $m$-th order homogeneous partial differential operator with constant coefficients and let $u$ be a smooth function defined in a neighbourhood of $\overline{P_{n}}$.

Assume that for each face $F$ of $P_{n}$ at least one of the following two conditions is satisfied:

(i) $F$ is characteristic for $L_{m}$;

(ii) $u$ satisfies in a neighbourhood of $F$ an equation $Q_{m-1}(u)=0$, and $F$ is noncharacteristic for $Q_{m-1}$, a homogeneous linear partial differential operator with constant coefficients of order $m-1$, which may depend on the face $F$.

Then for each codimension-2 face $P_{n-2}{ }^{i}$ of $P_{n}, i \in I$, there exists a homogeneous partial differential operator $L_{m-2}{ }^{i}$, of order $m-2$, with constant coefficients, such that:

$$
\int_{P_{n}} L_{m}(u) d x^{n}=\sum_{i \in I} \int_{P_{n-2}{ }^{i}} L_{m-2}^{i}(u) d x^{n-2} .
$$

The $L_{m-2}{ }^{i}$ depend only on $P_{n}, L_{m}$ and the $Q_{m-1}$.

For instance the Laplacian in Theorem 1 can be replaced by the wave operator, as long as $P_{n}$ is in general position with respect to the characteristic cone. A related general observation, well known for the wave equation, is contained in the following direct consequence of Stokes' theorem. 
Let $u$ be a smooth function defined in a neighbourhood of the unit cube $C_{n}=$ $\left\{x \in \mathbf{R}^{n} ; 0 \leq x_{j} \leq 1,1 \leq j \leq n\right\}$ and let $C_{0}=\left\{v_{k} ; 1 \leq k \leq 2^{n}\right\}$ be its vertices. Then:

$$
\int_{C_{n}} \partial_{1} \partial_{2} \ldots \partial_{n}(u)=\sum_{k=1}^{2^{n}} \epsilon_{k} u\left(v_{k}\right)
$$

where $\epsilon_{k}= \pm 1,1 \leq k \leq 2^{n}$.

Denoting by $\chi_{F}$ the characteristic function of a set $F$, the above result can equivalently be written in the sense of distributions:

$$
(-1)^{n} \partial_{1} \partial_{2} \ldots \partial_{n} \chi_{C_{n}}=\sum_{k} \epsilon_{k} \delta_{v_{k}}
$$

More generally, returning to an arbitrary polyhedron, we can restate Theorem 1 (with $\Delta$ replaced by a general second order operator $Q_{2}$ ) as follows.

Proposition 3. Let $L_{3}, Q_{2}$ be linear partial differential operators with constant coefficients of order 3, respectively 2. Then, for solutions $u$ of the equation $Q_{2}(u)=$ 0 in a neighbourhood of $\overline{P_{n}}$, the integral $\int_{P_{n}} L_{3}(u) d x^{n}$ can be pushed down to the $(n-2)$-skeleton $P_{n-2}$ of $P_{n}$ if and only if there are distributions $\sigma, \tau \in \mathcal{E}^{\prime}\left(\mathbf{R}^{n}\right)$ satisfying:

$$
L_{3}^{*} \chi_{P_{n}}=\sigma+Q_{2}^{*} \tau
$$

with

$$
\operatorname{supp}(\sigma) \subset \overline{P_{n-2}}, \quad \operatorname{supp}(\tau) \subset \overline{P_{n}} .
$$

Moreover, the structure of the distribution $\tau$ above turns out to be simple. More specifically, if $F_{i}, i \in I$, are the codimension-1 faces of $P_{n}$, then, up to functions in the kernel of $Q_{2}^{*}, \tau=\sum_{i \in I} a_{i} \delta_{F_{i}}$, with some constants $a_{i}$.

Indeed, assuming that the face $F_{i}$ is given by the equation $x_{n}=0$ we infer that in a neighbourhood of $F_{i}$ :

$$
L_{3}^{*} \chi_{P_{n}}(x)=C \partial_{n}^{3} H\left(x_{n}\right)=C \delta^{\prime \prime}\left(x_{n}\right)
$$

for some constant $C$, and where $H$ is the Heaviside function and $\delta$ the Dirac measure. Thus, locally on the face $F_{i}$, we have $\tau(x)=c \delta\left(x_{n}\right)$, with $c$ a constant.

The next result is somewhat analogous to example (2), but with the ordinary cube in $\mathbf{R}^{n}$ replaced by a "polycube" (or rather "poly-polygon") in $\mathbf{C}^{n}$. We denote $z=\left(z_{1}, \ldots, z_{n}\right) \in \mathbf{C}^{n}$, and $z_{j}=x_{j}+\sqrt{ }(-1) y_{j}, 1 \leq j \leq n$.

Theorem 4. Let $P_{2 n} \subset \mathbf{C}^{n}$ be a product of polygons in $\mathbf{C}$, and let $L_{n}=\partial / \partial z_{1} \ldots$ $\partial / \partial z_{n}$. Then there exist constants $\gamma_{v} \in \mathbf{C}$, where $v \in P_{0}$, so that for every analytic function $f$ defined in a neighbourhood of $\overline{P_{2 n}}$ :

$$
\int_{P_{2 n}} L_{n}^{2}(f) d x^{2 n}=\sum_{v \in P_{0}} \gamma_{v} f(v) .
$$

The real valued version of Theorem 4 reads then as follows.

Corollary 5. In the conditions of Theorem 4 , let $L_{3}{ }^{j}$ be homogeneous, constant coefficients, partial differential operators of order three in $x_{j}, y_{j}, 1 \leq j \leq n$. Then there are vectors $\gamma_{v}$ with $2^{n}$ components such that for every pluriharmonic function defined in a neighbourhood of $\overline{P_{2 n}}$ :

$$
\int_{P_{2 n}} L_{3}{ }^{1} L_{3}{ }^{2} \ldots L_{3}^{n}(u) d x^{2 n}=\left.\sum_{v \in P_{0}} \gamma_{v} \cdot \nabla_{\left(x_{1}, y_{1}\right)} \nabla_{\left(x_{2}, y_{2}\right)} \ldots \nabla_{\left(x_{n}, y_{n}\right)}(u)\right|_{v}
$$


Pluriharmonic functions in $\mathbf{C}^{n}$ are functions which are harmonic when restricted to any complex linear subspace. They arise as real parts of complex analytic functions (see [5] and the references cited there for details).

\section{Proofs}

Proof of Theorem 1. Although Theorem 1 is a consequence of Theorem 2, we include below a separate simple proof for better illustrating the main idea.

We shall first push the integral in the left member of (1) out to $\partial P_{n}$ and then further push the contribution from any given $(n-1)$-face $F \subset \partial P_{n}$ to $\partial F$. We may assume that the face $F$ is contained in the hyperplane $x_{n}=0$. The operator $L_{3}$ is a sum of monomials, so we can assume that $L_{3}=\partial_{i} \partial_{j} \partial_{k}$, with $i, j, k \in\{1,2, \ldots, n\}$, not necessarily distinct.

By using Stokes' theorem we are led to evaluate, up to a sign:

$$
\int_{\partial P_{n}} \partial_{i} \partial_{j}(u) d x_{1} \wedge \ldots \wedge d \hat{x}_{k} \wedge \ldots \wedge d x_{n} .
$$

The contribution from the face $F$ vanishes if $k \neq n$ (because $d x_{n}=0$ along $F$ ) and equals

if $k=n$.

$$
\int_{F} \partial_{i} \partial_{j}(u) d x_{1} \wedge \ldots \wedge d x_{n-1}
$$

In the case $i=j=n$ we can use Laplace's equation and write:

$$
\partial_{n}{ }^{2}(u)=-\sum_{k=1}^{n-1} \partial_{k}^{2}(u) .
$$

Therefore, we can assume that at least one of the indices $i, j$, say $j$, is different from $n$. Consequently, by Stokes' theorem again, the integral over $F$ reduces to (up to a sign):

$$
\int_{\partial F} \partial_{i}(u) d x_{1} \wedge \ldots \wedge \hat{x}_{j} \wedge \ldots \wedge d x_{n-1}
$$

and this proves Theorem 1 .

Proof of Theorem 2. We have, for arbitrary smooth functions $u$,

$$
L_{m}(u) d x_{1} \wedge \ldots \wedge d x_{n}=d \omega
$$

where

$$
\omega=\sum_{k=1}^{n} L_{m-1}^{k}(u) d x_{1} \wedge \ldots \wedge d \hat{x}_{k} \wedge \ldots \wedge d x_{n},
$$

for suitable $(m-1)$-th order operators $L_{m-1}{ }^{k}$ satisfying:

$$
\sum_{k=1}^{n}(-1)^{k-1} L_{m-1}^{k}\left(\partial_{k} u\right)=L_{m}(u) .
$$

Hence

$$
\int_{P_{n}} L_{m}(u) d x^{n}=\int_{\partial P_{n}} \omega
$$

Consider a face $F \subset \partial P_{n}$. We may assume that it is given by the equation $x_{n}=0$. Then

$$
\int_{F} \omega=\int_{F} L_{m-1}^{n}(u) d x_{1} \wedge \ldots \wedge d x_{n-1} .
$$


Case 1. Assume that $F$ is characteristic for $L_{m}$. This means that $L_{m}(u)$ does not contain the derivative $\partial_{n}^{m} u$. By relation $(5), L_{m-1}{ }^{n}(u)$ then does not contain $\partial_{n}^{m-1} u$. Thus every term of $L_{m}{ }^{n}$ contains a derivative $\partial_{j}$ with $j \neq n$. Hence the integrand in (6) is exact and the integral can be pushed to $\partial F$ (with no assumption on $u$ ).

Case 2. Assume that $Q_{m-1}(u)=0$ and $F$ is non-characteristic for $Q_{m-1}$. The latter means that $Q_{m-1}(u)$ contains $\partial_{n}^{m-1} u$. Thus, if $\partial_{n}^{m-1} u$ occurs in $L_{m-1}{ }^{n}(u)$, then it can be removed, and we can continue as in case 1 .

This finishes the proof of Theorem 2 .

Proof of Proposition 3. If formula (3) holds, then for $u$ satisfying $Q_{2}(u)=0$ in a neighbourhood of $\overline{P_{n}}$ we have:

$$
\begin{aligned}
\left\langle\chi_{P_{n}}, L_{3} u\right\rangle & =\left\langle L_{3}{ }^{*} \chi_{P_{n}}, u\right\rangle=\langle\sigma, u\rangle+\left\langle Q_{2}{ }^{*} \tau, u\right\rangle \\
& =\langle\sigma, u\rangle+\left\langle\tau, Q_{2} u\right\rangle=\langle\sigma, u\rangle,
\end{aligned}
$$

i.e., the integral of $L_{3} u$ over $P_{n}$ is pushed to the $(n-2)$-skeleton.

Conversely, suppose that

$$
\left\langle\chi_{P_{n}}, L_{3} u\right\rangle=\langle\sigma, u\rangle,
$$

for all $u$ satisfying $Q_{2}(u)=0$ in a neighbourhood of $\overline{P_{n}}$, with $\operatorname{supp}(\sigma) \subset \overline{P_{n-2}}$. Then the linear map

$$
Q_{2}(u) \longrightarrow\left\langle\chi_{P_{n}}, L_{3} u\right\rangle-\langle\sigma, u\rangle
$$

is well defined and continuous in the topology of $\mathcal{E}\left(\mathbf{R}^{n}\right)$. So, by Hahn-Banach Theorem, there exists a distribution $\tau$ with $\operatorname{supp}(\tau) \subset \overline{P_{n}}$, satisfying

$$
\left\langle\tau, Q_{2}(u)\right\rangle=\left\langle\chi_{P_{n}}, L_{3}(u)\right\rangle-\langle\sigma, u\rangle,
$$

or

$$
\left\langle Q_{2}{ }^{*} \tau, u\right\rangle=\left\langle L_{3}{ }^{*} \chi_{P_{n}}, u\right\rangle-\langle\sigma, u\rangle,
$$

for all smooth functions $u$ defined in a neighbourhood of $\overline{P_{n}}$.

Proof of Theorem 4. Let $P_{2 n}=\Delta_{1} \times \ldots \times \Delta_{n}$ be a product of planar polygons, and let $f\left(z_{1}, z_{2}, \ldots, z_{n}\right)$ be an analytic function in a neighbourhood of $\overline{P_{2 n}}$. Fix the variables $z_{j}=a_{j} \in \Delta_{j}, 2 \leq j \leq n$. Then the function $g\left(z_{1}\right)=f\left(z_{1}, a_{2}, \ldots, a_{n}\right)$ is analytic in a neighbourhood of $\overline{\Delta_{1}}$. By the Motzkin-Schoenberg-Davis theorem there are constants $c_{v} \in \mathbf{C}$, depending only on $\Delta_{1}$ and labelled by the vertices $v$ of $\Delta_{1}$, so that:

$$
\int_{\Delta_{1}} \frac{\partial^{2}(g)}{\partial z_{1}^{2}} d \overline{z_{1}} \wedge d z_{1}=\sum_{v \in\left(\Delta_{1}\right)_{0}} c_{v} g(v)
$$

An iterated integration then finishes the proof. The proof of Corollary 5 is entirely similar.

\section{REFERENCES}

[1] Davis, Ph., Triangle formulas in the complex plane, Math. Comp. 18(1964), 569-577. MR 29:4874

[2] Milanfar, P., Verghese, G.C., Karl, W.C., Wilsky, A.S., Reconstructing polygons from moments with connections to array processing, IEEE Trans. Signal Proc. 43(1995), 432- 443.

[3] Golub, G.H., Milanfar, P., Varah, J., A stable numerical method for inversting shape from moments, preprint 1997.

[4] Gustafsson, B., On mother bodies of convex polyhedra, SIAM J. Math. Anal. 29:5(1998). CMP 98:11 
[5] Henkin, G.M., Leiterer, J., Theory of functions on complex manifolds, Birkhäuser, Basel et al., 1984. MR 86i:32004

[6] Hörmander, L., The analysis of linear partial differential operators .I, Springer-Verlag, Berlin et al., 1983. MR 85g:35002a

[7] Siegel, D., Integration of harmonic functions over polygons, manuscript.

Department of Mathematics, Royal Institute of Technology, S-10044 Stockholm, SWEDEN

E-mail address: gbjorn@math.kth.se

Department of Mathematics, University of California, Santa Barbara, California 93106

E-mail address: mputinar@math.ucsb.edu 